\title{
The Effect of Swelling on the Elasticity of Polyisobutylene Networks at High Elongation*
}

\author{
Ramesh R. RAHALKAR and James E. MARK \\ Department of Chemistry and the Polymer Research Center \\ University of Cincinnati, Cincinnati, Ohio 45221, U.S.A.
}

(Received March 14, 1978)

\begin{abstract}
Sulfur-cured, unfilled networks of butyl rubber (essentially polyisobutylene, PIB) were swollen to varying degrees with a nonvolatile diluent. A study in elongation of their rupture points was carried out at sufficiently low temperature to permit strain-induced crystallization in the unswollen state. The stress-strain isotherms thus obtained differed somewhat from those similarly obtained for crystallizable networks of cis-1, 4-polybutadiene, in that for example the strain-induced crystallization never caused an abrupt decrease in the modulus. The marked increases in modulus which did occur at high elongations in the case of the unswollen PIB networks were found to diminish, and eventually to be suppressed entirely, with increased swelling. Both the ultimate strength and maximum extensibility decreased substantially with an increase in swelling. The fractional decreases in both quantities were much larger than for typical noncrystallizable networks of poly(dimethylsiloxane), but the ultimate strength was found to be much more sensitive to the reinforcing effect of the straininduced crystallization.
\end{abstract}

KEY WORDS Polyisobutylene / Butyl Rubber / Network Swelling / Stress-Strain Isotherms / Mooney-Rivlin Constants / Ultimate Properties / Strain-Induced Crystallization /

Strain-induced crystallization is of profound importance with regard to the elastomeric properties of unfilled polymer networks. ${ }^{1-5}$ The crystallites thus formed in the deformation process act as both physical crosslinks and nondeformable "filler particles," thereby greatly increasing the ultimate strength and maximum extensibility of the network. Unfilled networks incapable of such self-reinforcement remain weak, as is evidenced by the relatively low values of their ultimate properties. Correspondingly, any change in the experimental conditions which decreases the amount of straininduced crystallization attainable in a crystallizable network diminishes both its ultimate strength and maximum extensibility.

A number of studies ${ }^{6-11}$ have recently been carried out to provide a quantitative characterization of the effects cited above. The noncrystallizable

\footnotetext{
* Presented in part at the 26th International Congress of Pure and Applied Chemistry, Tokyo, Japan, September, 1977.
}

networks were prepared from poly(dimethylsiloxane) (PDMS) ${ }^{6,8}$ and from atactic poly(ethyl acrylate) ${ }^{6}$ and the crystallizable ones from polyisobutylene (PIB, or "butyl rubber") ${ }^{7}$ and cis-1, 4polybutadiene ( cis-PBD). ${ }^{9,10}$ The networks were studied in elongation up to rupture points as a function of (i) the method of crosslinking, ${ }^{9,10}$ (ii) crosslink density, ${ }^{6-10}$ (iii) temperature, ${ }^{7,9,10}$ and (iv) degree of swelling. ${ }^{8,10}$ The type of information obtained in such studies is obviously of great fundamental and practical interest and, for this reason, the present investigation applies such measurements to crystallizable networks of PIB. The primary goal is the characterization of the effect of swelling on the ultimate properties of PIB networks, and the comparison of these results with the previously published results obtained on PDMS networks and on cis-PBD networks.

\section{EXPERIMENTAL}

The networks employed, PIB-1 through 3, were 
obtained from the same sheets which had been sulfur-cured for a previous study $^{7}$ of PIB networks. ${ }^{12}$ Briefly, they were obtained from mixtures of butyl rubber, with sulfur, zinc oxide, stearic acid, and two organic disulfides. The mixtures were pressed into aluminum molds, sealed, and then cross-linked by heating to $150^{\circ} \mathrm{C}$ in a vacuum oven for $1 \mathrm{~h}$. They were purified (extracted) exactly as described elsewhere, ${ }^{7}$ and a number of strips were cut from each of the three samples by suitable rubber dies. Strip dimensions were of the order of $0.1 \times 0.2 \times 2 \mathrm{~cm}$. The temperatures for the investigation were chosen so as to give significant strain-induced crystallization in these networks in the unswollen state $;^{7}$ for PIB-1, 2, 3 these were 30,20 , and $15^{\circ} \mathrm{C}$, respectively. The diluent employed was 1,2,4-trichlorobenzene, and the degree of swelling was adjusted to give values of the volume fraction $v_{2}$ of polymer in the network of $1.00,0.80,0.60$, and 0.40 .

Stress-strain isotherms were obtained on the networks, unswollen and swollen, using the standard techniques described in detail elsewhere. ${ }^{6-10}$ Since the measurements were carried out up to the rupture points, each degree of swelling of a sample required a separate network strip. The rupture was observed to occur generally in the vicinity of the clamped regions of the sample strips.

\section{RESULTS AND DISCUSSION}

The stress-strain data were treated in terms of the reduced stress or modulus defined by ${ }^{13-16}$

$$
\left[f^{*}\right] \equiv f v_{2}^{1 / 3} /\left[A^{*}\left(\alpha-\alpha^{-2}\right)\right]
$$

where $f$ is the elastic force, and $A^{*}$ is the unswollen, unstretched cross-sectional area of the sample strip. The elongation $\alpha$ is given by $L / L_{i}$, where $L$ is the length of the sample in the stretched state and $L_{i}$ is its length unstretched at the same value of $v_{2}$. The values of $\left[f^{*}\right]$ thus calculated were represented using the semi-empirical equation of Mooney and Rivlin ${ }^{17}$

$$
\left[f^{*}\right]=2 C_{1}+2 C_{2} \alpha^{-1}
$$

in which $2 C_{1}$ and $2 C_{2}$ are constants independent of $\alpha$, with the latter serving as a measure of the extent to which an observed isotherm departs from the form predicted by the simplest molecular theories of rubber-like elasticity. ${ }^{2,3,16}$ The resulting plots of reduced stress against reciprocal elonga-

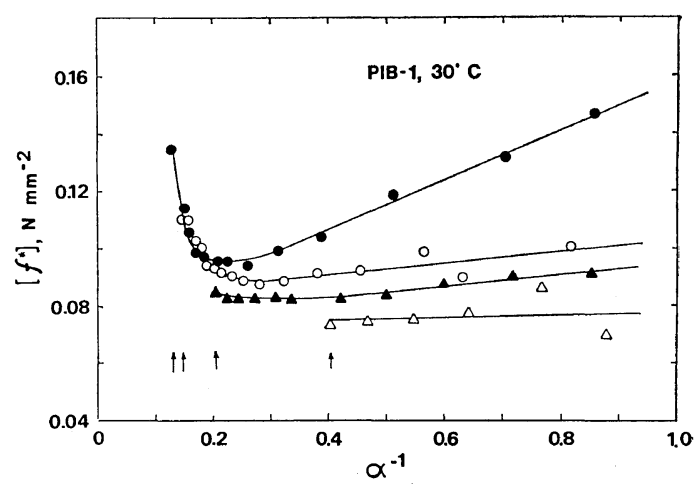

Figure 1. Stress-strain isotherms for polyisobutylene network PIB-1 studied in both the unswollen and swollen states at $30^{\circ} \mathrm{C}$. The following information pertains to both this figure and the following two figures. Values chosen for the volume fraction $v_{2}$ of polymer in each of the networks: $1.00(\bullet), 0.80(\bigcirc)$, $0.60(\Delta)$, and $0.40(\triangle)$; the diluent employed was 1,2 , 4-trichlorobenzene. The arrows locate the values of the elongation $\alpha$ at which the rupture occurred.

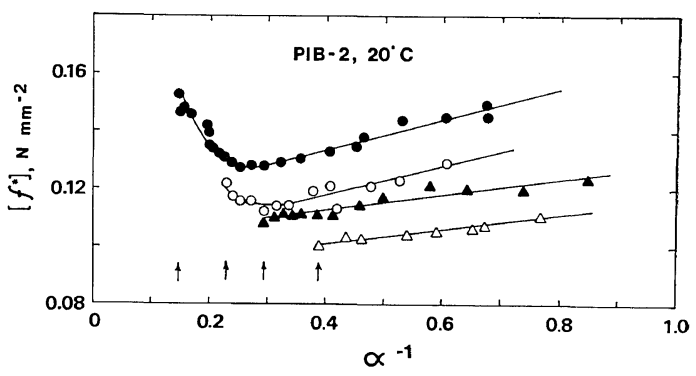

Figure 2. Stress-strain isotherms for network PIB2 at $20^{\circ} \mathrm{C}$; see legend to Figure 1 .

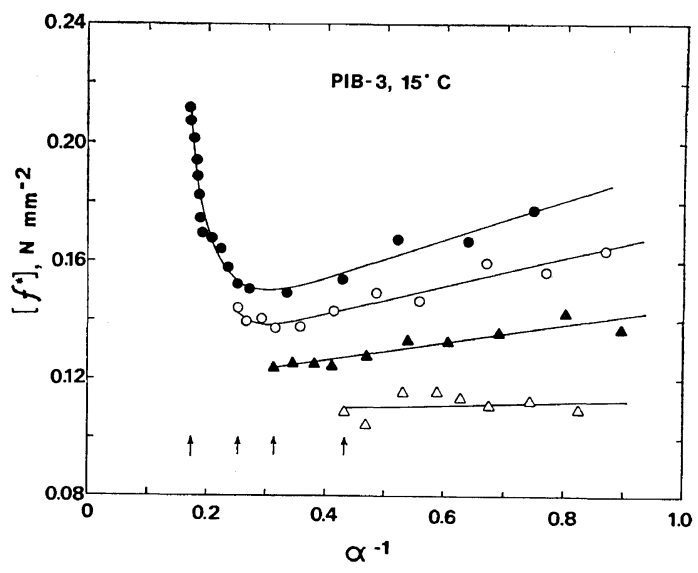

Figure 3. Stress-strain isotherms for network PIB3 at $15^{\circ} \mathrm{C}$; see legend to Figure 1 . 
The Effect of Swelling on the Elasticity of Polyisobutylene Networks at High Elongation

Table I. Mooney-Rivlin constants and ultimate properties

\begin{tabular}{|c|c|c|c|c|c|c|c|}
\hline \multirow{2}{*}{ System } & \multirow{2}{*}{$v_{2}$} & \multirow{2}{*}{$\begin{array}{c}2 C_{1}, \\
\mathrm{~N} \mathrm{~mm}^{-2}\end{array}$} & \multirow{2}{*}{$\begin{array}{c}2 C_{2,} \\
\mathrm{~N} \mathrm{~mm}^{-2}\end{array}$} & \multirow{2}{*}{$\begin{array}{c}f_{\mathrm{r}}^{*}, \\
\mathrm{Nm}\end{array}$} & \multirow{2}{*}{$\frac{f_{\mathrm{r}}^{*}\left(v_{2}\right)}{f_{\mathrm{r}}^{*}(1.0)}$} & \multirow{2}{*}{$\lambda_{r}$} & \multirow{2}{*}{$\begin{array}{l}\lambda_{\mathrm{r}}\left(v_{2}\right) \\
\lambda_{\mathrm{r}}(1.0)\end{array}$} \\
\hline & & & & & & & \\
\hline \multirow[t]{4}{*}{ PIB- $1,30^{\circ} \mathrm{C}$} & 1.00 & 0.0722 & 0.0864 & 1.04 & 1.000 & 7.69 & 1.000 \\
\hline & 0.80 & 0.0833 & 0.0197 & 0.794 & 0.766 & 7.18 & 0.934 \\
\hline & 0.60 & 0.0743 & 0.0210 & 0.487 & 0.470 & 5.78 & 0.752 \\
\hline & 0.40 & 0.0734 & 0.0043 & 0.232 & 0.224 & 3.39 & 0.441 \\
\hline \multirow[t]{4}{*}{ PIB-2, $20^{\circ} \mathrm{C}$} & 1.00 & 0.113 & 0.0524 & 1.02 & 1.000 & 6.67 & 1.000 \\
\hline & 0.80 & 0.0976 & 0.0503 & 0.564 & 0.555 & 4.68 & 0.703 \\
\hline & 0.60 & 0.104 & 0.0271 & 0.428 & 0.421 & 3.97 & 0.595 \\
\hline & 0.40 & 0.0905 & 0.0248 & 0.332 & 0.326 & 3.53 & 0.529 \\
\hline \multirow[t]{4}{*}{ PIB- $3,15^{\circ} \mathrm{C}$} & 1.00 & 0.128 & 0.0661 & 1.24 & 1.000 & 5.88 & 1.000 \\
\hline & 0.80 & 0.123 & 0.0473 & 0.611 & 0.492 & 4.31 & 0.733 \\
\hline & 0.60 & 0.114 & 0.0305 & 0.441 & 0.356 & 3.71 & 0.630 \\
\hline & 0.40 & 0.108 & 0.0058 & 0.320 & 0.258 & 3.16 & 0.537 \\
\hline
\end{tabular}

tion are shown in Figures $1-3$. Least-squares analysis was used to locate the straight lines representing the linear portions of the isotherms. The resulting values of the intercept and slope, $2 C_{1}$ and $2 C_{2}$, respectively, are given in the third and fourth columns of Table I.

The constant $2 C_{1}$ is related to the network structure by the equation ${ }^{2,10,18-20}$

$$
2 C_{1}=C \rho k T / M_{\mathrm{c}}
$$

where $C$ is a constant equal to one-half (according to the most recent and reliable theoretical and experimental investigations), ${ }^{18-21} \rho$ is the density of the network, $k$ is the Boltzmann constant, $T$ is the absolute temperature, and $M_{\mathrm{c}}$ is the molecular weight between crosslinks. As shown in Table I, the values of $2 C_{1}$ are, to a very good approximation, independent of the degree of swelling, as expected from eq 3. Values of $10^{-3} M_{\mathrm{c}}$ calculated from this equation are $15.5( \pm 0.7), 11.7$ ( \pm 0.8$)$, and $9.54( \pm 0.59)$ for PIB-1 through 3, respectively. These results (averaged over different degrees of swelling) are in close agreement with previous results obtained from stress-strain measurements on the same networks carried out as a function of temperature in the unswollen state. ${ }^{7}$ These earlier estimates of $10^{-3} M_{\mathrm{c}}$ utilized a value of $C$ of unity rather than the value of one-half now known to be the more nearly correct, and therefore used in the present study. ${ }^{18-20}$ The earlier results, thus revised to permit comparisons with the present results, give values of $M_{\mathrm{c}}$ (averaged over different temperatures) of 15.5 ( \pm 0.7$), 12.3( \pm 0.8)$, and $11.2( \pm 0.2)$, respectively.
The constant $2 C_{2}$ is seen to decrease markedly with an increase in degree of swelling (decrease in $v_{2}$ ). Such a decrease has been found for virtually all polymer networks thus studied in both the unswollen and swollen states. ${ }^{16}$ It has recently been interpreted in terms of the diluent's effect on the firmness with which the crosslinks are embedded in the network medium, which in turn affects the extent to which the network deformation will be affine. ${ }^{18-20}$

The nonlinear portions of the isotherms are indicative of strain-induced crystallization. ${ }^{2,6-10,22-24}$ It is of interest to note that the nonlinearity in the case of the PIB networks is always in the direction of an increase in the reduced stress. This is rather different from the case of some of the cis-PBD networks ${ }^{9,10}$ (and some natural rubber networks), ${ }^{25}$ which frequently show a downturn in $\left[f^{*}\right]$ prior to its increase. Such a decrease in $\left[f^{*}\right]$ is due to the fact that the chain sequences within the crystallites are in regular, extended conformations and the crystallites are themselves oriented along the axis of stretching. ${ }^{9,10,26-28}$ The straightening and aligning of portions of the network chains thus tends to decrease the deformation in the remaining portions. In the PIB networks, this effect apparently does not predominate over the increase in the degree of crosslinking resulting from the fact that the formed crystallites act as multifunctional crosslinks. The difference in behavior between PIB and cis-PBD is not really surprising since different polymers generally have different melting points, 
heats of fusion, rates of crystallization, crystallinestate configurations, crystalline morphologies, etc., which could all affect the way the straininduced crystallization affects the reduced stress.

One measure of the reinforcing effect of the crystallites on the network structure is the ultimate strength, which can be represented in terms of the nominal or engineering stress $f^{*} \equiv f \mid A^{*}$. Its values $f_{\mathrm{r}}^{*}$ at rupture were obtained from the curves of Figures $1-3$, in which the corresponding values of the elongation at rupture are specified by the short vertical arrows. The results are given in column five of the table. The effect of swelling is most conveniently characterized through the ratio $f_{\mathbf{r}}^{*}\left(v_{2}\right) / f_{\mathrm{r}}^{*}(1.0)$ of the ultimate strength relative to its value for the unswollen network. The values thus calculated are given in the following column, and are shown as a function of the degree of swelling in Figure 4. Each point represents the average value

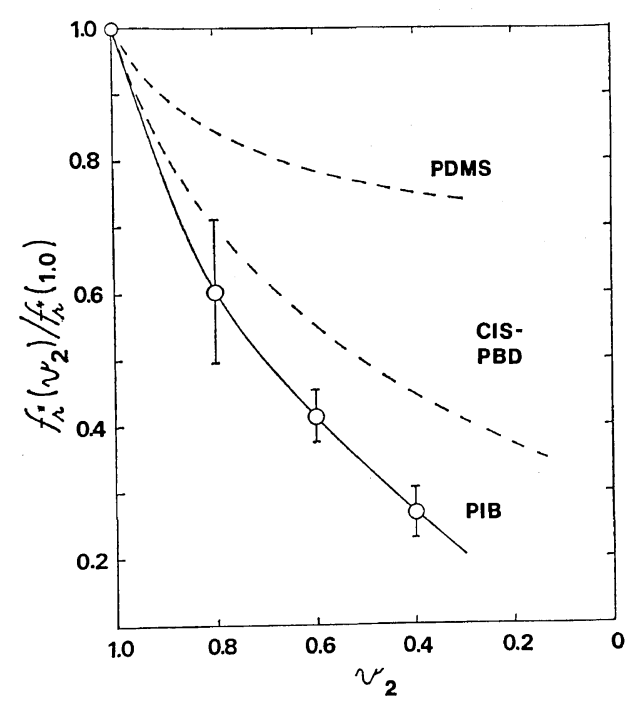

Figure 4. The effect of swelling on the average ultimate strength (expressed relative to the values obtained in the unswollen state) for the PIB networks. Included for purposes of comparison in this and the following figure are analogous results obtained for a typical noncrystallizable network of poly(dimethylsiloxane $)^{8}$ and a typical crystallizable network of cis1, 4-polybutadiene. ${ }^{10}$

for the three PIB networks, and the error bars give the average deviation from each of these plotted values. Included for purposes of comparison are the curves obtained from the previous results reported for networks of $\mathrm{PDMS}^{8}$ and cis-PBD. ${ }^{10}$ The diluent depresses the melting point of the PIB chains, thus diminishing the amount of straininduced crystallization. The ultimate strength of the PIB networks thus decreases much more markedly with swelling than it does in PDMS, as was observed. ${ }^{8}$ This decrease is also larger than that found for $c i s-\mathrm{PBD}$, which is of considerable interest since an increase in temperature also causes a larger decrease in the ultimate strength and maximum extensibility in the case of PIB., ${ }^{7,9}$ Of possible importance is the fact that, although PIB and cis-PBD have very similar melting points $\left(5^{29}\right.$ and $1^{\circ} \mathrm{C},{ }^{30,31}$ respectively), they have rather different values for the heat of fusion $\Delta H_{\mathrm{m}}$. The values for PIB and cis-PBD are $2.8^{32}$ and $2.20^{30}$ $\mathrm{kcal}$ per mole of repeat units, respectively, which correspond to 1.4 and $0.55 \mathrm{kcal}$ per mole of skeletal bonds. Since the extent to which the melting point of a network is increased by elongation depends inversely on the heat of fusion, ${ }^{2,26,33}$ the larger value of $\Delta H_{\mathrm{m}}$ for PIB could account in part for its larger decrease in ultimate strength upon an increase in temperature. The larger decrease with an increase in the degree of swelling admits of the same rationalization, but may be complicated by the fact that the larger value of $\Delta H_{\mathrm{m}}$ for PIB should also decrease the extent to which the melting point is depressed by the diluent. ${ }^{2,33,34}$

Another measure of the reinforcing effect of the strain-induced crystallization is the elongation $\alpha_{r}$ at rupture, corrected to account for the fact that the chains in a swollen network are already stretched by the presence of the diluent prior to further deformation in the elongation process. Values of the corrected or total elongation ${ }^{8} \lambda_{\mathrm{r}}=\alpha_{\mathrm{r}} v_{2}{ }^{-1 / 3}$ are given in column seven of the Table. The ratio $\lambda_{\mathrm{r}}\left(v_{2}\right) / \lambda_{\mathrm{r}}(1.0)$ of the maximum extensibility relative to its value in the unswollen state is given in the final column, and is plotted as a function of $v_{2}$ in Figure 5. Again, because of the decrease in the strain-induced crystallization, the diluent causes a much larger decrease in this ratio in the case of the PIB than it does in the case of PDMS. ${ }^{8}$ The decrease in maximum extensibility observed for PIB is very similar to that for cis-PBD, ${ }^{10}$ however, thus suggesting that the ultimate strength of a network is generally much more enhanced by strain-induced crystallization than is the maximum extensibility. This is due to the fact, illustrated 
The Effect of Swelling on the Elasticity of Polyisobutylene Networks at High Elongation

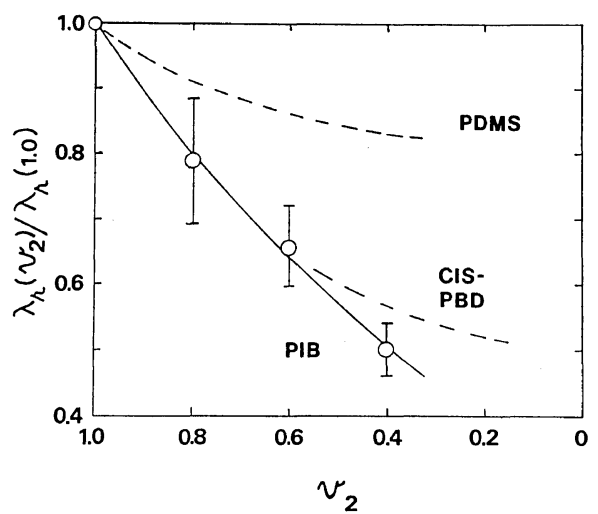

Figure 5. The effect of swelling on the total elongation to rupture; see legend to Figure 4.

here in Figures 1 and 3, that once significant amounts of crystallization are induced, $\left[f^{*}\right]$ and $f^{*}$ generally increase nearly vertically, i.e., very markedly with little increase in elongation. ${ }^{7,9,10}$ Additional strain-induced crystallization thus continues to increase the strength of the material at very nearly the same value of the elongation, and as a result, the maximum extensibility remains very nearly constant. The ultimate strength should therefore probably be preferred in general in characterizing the effects of strain-induced crystallization on the elastomeric properties of a polymer network at very high elongation.

Acknowledgment. It is a pleasure to acknowledge, with gratitude, the financial support provided by the National Science Foundation through Grant DMR 77-18356 (Polymers Program, Division of Materials Research).

\section{REFERENCES}

1. G. Gee, J. Polym. Sci., 2, 451 (1947).

2. P. J. Flory, "Principles of Polymer Chemistry," Cornell University Press, Ithaca, N. Y., 1953.

3. L. R. G. Treloar, "The Physics of Rubber Elasticity," 2nd ed., Clarendon Press, Oxford, 1958; 3rd ed., 1975.

4. T. L. Smith, Polym. Eng. Sci., 17, 129 (1977).

5. J. E. Mark, Polym. Eng. Sci., 18, in press. (1979).

6. J. E. Mark, M. Kato, and J. H. Ko, J. Polym. Sci., Part C, No. 54, 217 (1976).

7. M. Kato and J. E. Mark, Rubber Chem. Technol.,
49, 85 (1976).

8. D. S. Chiu and J. E. Mark, Colloid Polym. Sci., 255, 644 (1977).

9. T.-K. Su and J. E. Mark, Macromolecules, 10, 120 (1977).

10. D. S. Chiu, T.-K. Su, and J. E. Mark, Macromolecules, 10, 1110 (1977).

11. J. E. Mark, manuscript submitted to Polym. Eng. Sci.; see also, Polym. Prepr., 19 (1), 327 (1978).

12. Mixtures of butyl rubber with suitable curing agents were generously supplied by F. P. Baldwin of the Exxon Chemical Company.

13. A. Ciferri and P. J. Flory, J. Appl. Phys., 30, 1498, (1959).

14. J. E. Mark and P. J. Flory, J. Appl. Phys., 37 4635 (1966).

15. P. J. Flory and Y. Tatara, J. Polym. Sci., Polym. Phys. Ed., 13, 683 (1975).

16. J. E. Mark, Rubber Chem. Technol., 48, 495 (1975), and pertinent references cited therein.

17. M. Mooney, J. Appl. Phys., 19, 434 (1948): R. S. Rivlin, Phil. Trans. R. Soc. London, Ser. A, 241, 379 (1948).

18. P. J. Flory, Proc. R. Soc. London, Ser. A., 351, 351 (1976).

19. J. E. Mark and J. L. Sullivan, J. Chem. Phys., 66, 1006 (1977).

20. P. J. Flory, J. Chem. Phys., 66, 5720 (1977).

21. H. M. James and E. Guth, J. Chem. Phys., 15, 669 (1947).

22. P. J. Flory, Chem. Rev., 35, 51 (1944).

23. P. J. Flory, Ind. Eng. Chem., 38, 417 (1946).

24. T. G Fox, Jr., P. J. Flory, and R. E. Marshall, J. Chem. Phys., 17, 704 (1949).

25. K. J. Smith, Jr., A. Greene, and A. Ciferri, Kolloid Z. Z. Polym., 194, 49 (1964).

26. P. J. Flory, J. Chem. Phys., 15, 397 (1947).

27. P. J. Flory, Science, 124, 53 (1956).

28. A. N. Gent, J. Polym. Sci., Part A-2, 3, 3787 (1965); 4, 447 (1966).

29. R. M. Kell, B. Bennett, and P. B. Stickney, Rubber Chem. Technol., 31, 499 (1958).

30. G. Natta and G. Moraglio, Makromol. Chem., 66, 218 (1963).

31. J. Brandrup and E. H. Immergut, Ed., "Polymer Handbook," 2d ed., Wiley-Interscience, New York, N. Y., 1975.

32. J. R. Schaefgen, J. Polym. Sci., 38, 549 (1959).

33. L. Mandelkern, "Crystallization of Polymers," McGraw-Hill, New York, N. Y., 1964.

34. P. J. Flory, J. Chem. Phys., 15, 684 (1947); 17, 223 (1949). 